\title{
Components of events and processes
}

\author{
Xu Xu \\ Pennsylvania State University, Harrisburg, Pennsylvania \\ AND \\ Katja Wiemer-Hastings, Joseph P. Magliano, and Betty Birner \\ Northern Illinois University, DeKalb, Illinois
}

\begin{abstract}
Rips and Estin (1998) provided evidence that mental events such as dreaming are more homogeneous than physical events such as checking out a book; that is, their parts are more difficult to distinguish. In their experiment, participants listed more distinctive properties for the parts of physical events than for the parts of mental events. However, the physicality of stimuli was confounded with temporal aspects. Mental stimuli tended to be processes, and physical stimuli, events. This study tested homogeneity with new stimuli separating out the factors of physicality and aspect. Consistently, both physicality and aspect had significant effects on the perceived homogeneity of activities, as measured by the number of listed parts, the number of distinctive properties of each part, and homogeneity ratings. The study shows that homogeneity is strongly influenced by aspect but that physicality remains a robust factor for homogeneity, even after taking aspect into account.
\end{abstract}

We experience and remember all kinds of events that unfold in time. Research on event structures provides insight into how people comprehend, associate, and organize information over time. For this reason, diverse event types have been investigated, including physical movements (e.g., Newtson, 1973), musical sequences (e.g., Boltz, 1998), film segments (e.g., Magliano, Miller, \& Zwaan, 2001), and routine events (Schank \& Abelson, 1977). The focus of most studies has been on physical events - that is, events with observable elements, such as motion, actions, objects, and people. In contrast, mental events have to date attracted little attention. Mental events evolve around nonobservable elements, such as knowledge, goals, and beliefs, and are an important aspect of daily experiences. As such, it is important to rectify this imbalance by systematically comparing the two types of events.

The temporal structure of events has been investigated extensively (for a detailed review, see Zacks \& Tversky, 2001). It is central to our understanding of how events are represented and processed, since their characteristics unfold in time. Thus, one step to improve our understanding of mental events is to compare the temporal structure of physical and mental events: Are they fundamentally the same, or are there important differences? Rips and Estin (1998) pointed to one important difference in the temporal structure of mental events - namely, that mental events tend to be more homogeneous than physical events. Homogeneous events tend to be difficult to break down into temporal phases. For example, a physical event such as having breakfast involves distinct steps such as making coffee, getting a bowl, pouring cereal and milk, eating the cereal, drinking the coffee, and cleaning up (Schank \& Abelson, 1977). In contrast, the salient components of mental events are goals and thinking processes, which cannot be easily analyzed further into distinct steps. For example, deciding involves an evaluation and a thought process, but these processes do not seem to occur sequentially.

As a consequence, each part of a mental event tends to be quite similar to the overall event; indeed, people typically regard both the part and the whole as belonging to the same superordinate category (Rips \& Estin, 1998). For example, in Rips and Estin's study, reasoning is an instance of a mental event. Using logic is a part of reasoning. Participants indicated that both using logic and reasoning are kinds of thinking. In contrast, the part and the whole of an object were regarded as belonging to different superordinates. For example, a stem is a part of an apple. An apple is a kind of fruit, but a stem is not. Physical events appeared to be an intermediate case. Physical events and their parts were more likely to be cross-classified into the same superordinate than were objects and their parts, but less likely than were mental events and their parts.

To test the homogeneity of physical and mental events empirically, Rips and Estin (1998) first collected typical parts from participants for a number of physical and mental events. For example, organizing may be a part of the mental event of planning, and getting an ID a part of the physical event of checking out books. In the main experiment, participants read a typical part for each event, and then listed properties for this part that were common to or distinct from other parts of the event. Common properties were shared by all the other parts of that event; distinctive

X.Xu,xuxu@psu.edu 
properties were shared by no other parts of that event. The critical measure for event homogeneity was the number of distinctive properties listed for the parts. The parts of a homogeneous event should have few or no distinctive properties; the parts of a nonhomogeneous event should have many distinctive properties. Their results showed that parts of physical events were more distinct than those of mental events. This is consistent with the hypothesis that homogeneity differences may underlie the part-whole classification differences observed previously for mental versus physical events.

The relation between event homogeneity and partwhole classification seems straightforward. However, a closer examination of the stimuli used in the Rips and Estin (1998) study revealed that the difference in homogeneity might actually be due to a temporal aspect, rather than to physicality. Applying the distinction between events and processes provided by Vendler (1967), most of the stimuli used as "physical events" (e.g., checking out books, doing dishes, catching a plane) were truly events, but most of the "mental events" (e.g., dreaming, reading, imagining) would be more aptly classified as processes. This is critical, because differences in homogeneity could be explained by the definitions of event versus process. According to Vendler, events involve noticeable situational changes between time slices and have starting and ending points. In contrast, processes are characterized as continuous activities that last for an indefinite period of time and do not have any ending points. The moments within a process tend to be identical. Thus, differences in homogeneity might, to some extent, be due to aspect (i.e., event vs. process) rather than just to physicality (i.e., physical vs. mental). Events should have more parts than do processes, and the parts of an event should be more distinct than are the parts of a process.

Experiments $1 \mathrm{~A}$ and $1 \mathrm{~B}$ of the present study empirically tested the possibility that the homogeneity differences between the mental and physical "events" in Rips and Estin's (1998) materials was in part due to a systematic difference in aspect. Experiments 2, 3, and 4 compared the effects of both physicality and aspect on homogeneity with the help of stimuli that varied both in physicality (physical vs. mental) and in temporal aspect (event vs. process). These experiments aimed to test whether physicality would influence the homogeneity of activities, even after controlling for temporal aspect. To avoid confusion, we will henceforth refer to events and processes collectively as activities. By crossing physicality and aspect, we obtained four different types of activity: physical event, physical process, mental event, and mental process.

The tasks in Experiments 2, 3, and 4 were adapted from Rips and Estin's (1998) methodology. Experiment 2 investigated structural differences of the four different types of activity in terms of the numbers of temporal parts generated by participants. Experiment 3 examined the distinctiveness of the parts through common and distinctive property listings. Experiment 4 used ratings to compare how easy or difficult it is to distinguish the parts of the four types of activity. There are two possible outcomes to these experiments. First, if physical activities are less homogeneous than mental activities, there may be two main effects: mental activities may have fewer parts, parts of less distinctiveness, and parts with lower differentiation ratings than do physical activities; and processes may have fewer parts, parts of less distinctiveness, and parts with lower differentiation ratings than do events. Second, if Rips and Estin's findings were due exclusively to aspect, only the main effect of aspect would be found, with processes having fewer parts, parts of less distinctiveness, and parts of lower differentiation ratings than do events.

\section{EXPERIMENT 1A}

This experiment empirically tested the possibility that physicality and temporal aspect were confounded in the stimuli used by Rips and Estin (1998). Specifically, the experiment examined whether "mental events" from their study would be classified as processes more often than as events, and conversely, whether "physical events" would be classified more often as events than as processes.

\section{Method}

Participants. Twenty participants were recruited from undergraduate introductory psychology classes at Northern Illinois University. Students received course credit for their participation in this experiment.

Materials. The testing materials in Rips and Estin's (1998) study included three groups of stimuli: objects, physical events, and mental events. In this experiment, only physical and mental activities were used, with 12 items in each group (materials listed in Appendix A).

Procedure. The 24 terms were presented to half of the participants in random order. The other half of the participants received the items in the reverse order. Participants were instructed to indicate which type of activity, an event or a process, they thought each term referred to. Examples of events and processes were included in the instructions to help participants understand the task. The six examples were either directly taken from Vendler's (1967) study or constructed based on his arguments. Two examples of an event were jogging a mile and drawing a circle. Two examples of a process were playing piano and chatting with friends.

\section{Results and Discussion}

For each term, we calculated the proportion of participants who indicated the term as an event (e). Accordingly, the proportion of participants who indicated the term as a process was $p=1-e$. An independent two-sample $t$ test was performed on $e$ with the physicality distinction (physical vs. mental, as in Rips \& Estin, 1998) as the independent variable. For physical activities, $e$ was higher $(M=.738)$ than for mental activities $(M=.217)$. This difference was highly significant $[t(22)=12.692, p<$ $.001]$. Consistent with our impression, participants were more likely to regard the "physical events" as events, but more likely to regard the "mental events" as processes.

\section{EXPERIMENT 1B}

We were concerned that participants in Experiment 1A might have been coached by the examples for event and process provided in the instructions. Specifically, if people had obtained implicit criteria from the examples to complete the task, any distinction they drew between events and processes could have been a learning effect, 
and temporal aspect could not have been a factor affecting the responses in Rips and Estin's (1998) study. Therefore, the experiment was replicated without any examples in the instructions.

\section{Method}

Participants. Eighteen participants who had not participated in Experiment 1A were recruited from undergraduate introductory psychology classes at Northern Illinois University. Students received course credit for their participation in this experiment.

Materials. The materials were the same as in Experiment 1A.

Procedure. The 24 terms were presented to half of the participants in random order. The other half of the participants received items in the reverse order. Participants were simply instructed to indicate which type of activity, an event or a process, they thought each term referred to.

\section{Results and Discussion}

For each term, we calculated $e$, the proportion of participants who indicated the term as an event. An independent two-sample $t$ test was carried out on $e$. Again, physical activities were judged as events $(M=.667)$ significantly more often than were mental activities $(M=$ .232) $[t(22)=8.028, p<.001]$, suggesting that Rips and Estin's (1998) study compared mainly physical events and mental processes. It is not clear which of the two factors, physicality or aspect, was associated with the reported difference in homogeneity. The subsequent experiments tested these two factors separately.

\section{EXPERIMENT 2}

The purpose of this experiment was to investigate the temporal structures of different activities by comparing the number of temporal parts that participants generated for each of the four types of activities: mental events, mental processes, physical events, and physical processes. If physical activities are truly less homogeneous than mental activities, two main effects are predicted: First, consistent with Rips and Estin's (1998) findings, mental activities should have fewer parts than physical activities; second, given Vendler's (1967) definitions, processes should have fewer parts than do events.

\section{Method}

Participants. Twenty participants who did not take part in the previous experiments were recruited from undergraduate introductory psychology classes at NIU. Students received course credit for participation in this experiment.

Materials. The testing materials in Rips and Estin's (1998) study included both gerundive verbs (reading, dreaming, etc.) and phrases in the form of gerundive verbs with nouns (writing a letter, doing the dishes, etc.). According to Vendler (1967), differences in linguistic form suggest different aspect. Gerunds usually describe processes, whereas gerundive phrases imply the accomplishment of activities. For example, drawing is regarded as a process - that is, a continuous ongoing activity - whereas drawing a circle implies the accomplishment of the activity. To control for the effect of linguistic form, all stimuli in the present study were single nouns (e.g., imagination, interview). Seventy activity nouns were randomly sampled from the MRC Psycholinguistic Database 2.0, an online dictionary file provided as a public research resource. Activities were counted as mental activities if they involved a mental process as a central element; otherwise, they were counted as physical activities.
A norming study was conducted to identify events and processes among the 70 nouns. Four independent judges who were either professors or graduate students at Northern Illinois University were asked to classify the 70 nouns as events, processes, or states. The four judges were completely blind to the purpose and hypotheses of the present research. The activity nouns that were consistently classified by at least three out of four judges as events or processes were retained. Twelve events and 12 processes were selected by the first author from these terms. In each group, half of the activity terms were physical and half were mental, resulting in four groups of terms: six physical events, six physical processes, six mental events, and six mental processes. Table 1 lists the 24 words along with judges' agreement rate on each word; they can also be seen in Appendix B. These 24 nouns constituted the first set of stimuli.

For better comparability to Rips and Estin's (1998) study, a second set of stimuli was included that presented the same activities in the form of gerunds instead of nouns (e.g., interviewing instead of interview). This set included only 21 activity concepts out of the total 24 in the first set. Three nouns - déjà vu, concert, and funeral - were excluded because they could not be naturally transformed into gerunds.

Procedure. Participants were randomly assigned to the conditions with either noun or gerund terms, with 10 participants in each condition. The 24 terms in each condition were presented to participants in a booklet in random order, with one term at the top of each page. Participants were instructed to list temporal parts for each of the 24 activity terms. The instruction was similar to Rips and Estin's (1998) study, except that the word phase was used instead of part to refer to the temporal part of an activity. The purpose was to direct participants to focus on temporal structures of activity. The instruction referred to the 24 terms as common activities that could be divided into different phases along the temporal dimension. Participants were asked to write down as many phases as they could think of, but only the phases that were necessarily contained by all instances of that activity. Two example terms with some of their temporal parts were included to help participants understand the task. (Exam and evaluation were used as examples for the noun stimuli condition; sunbathing and evaluating were used for the gerund stimuli condition.) For example, evaluation was presented with the parts selecting a criterion; assessing how well the criterion is met; and making a judgment. All participants completed the task within 40 min.

\section{Results and Discussion}

The responses were evaluated before the numbers of parts were counted. Synonyms for, or descriptions of, different aspects of the same part of an activity were counted as the same part. For example, going faster than before and moving

Table 1

Judge Agreement (\%) on the Aspect (Event or Process) of Activities

\begin{tabular}{lllr}
\hline \multicolumn{1}{c}{ Event } & \multicolumn{2}{c}{ Process } \\
\hline Physical & & & \\
Funeral & 100 & Acceleration & 100 \\
Date & 100 & Investigation & 100 \\
Party & 100 & Development & 75 \\
Robbery & 100 & Exploration & 100 \\
Concert & 100 & Decomposition & 100 \\
Interview & 100 & Management & 75 \\
Mean & 100 & & 91.7 \\
Mental & & & \\
Déjà vu & 100 & Imagination & 75 \\
Judgment & 100 & Elaboration & 100 \\
Debate & 75 & Conceptualization & 75 \\
Recognition & 75 & Reflection & 75 \\
Approval & 75 & Contemplation & 75 \\
Realization & 75 & Recollection & 75 \\
Mean & 83.3 & & 79.2 \\
\hline
\end{tabular}


at a higher speed were counted as the same part listed for acceleration; picking up someone and being picked up by someone were counted as the same part listed for date. Nonresponses such as "this is a hard word" were not counted.

Activities presented as nouns. A 2 (physical vs. mental) $\times 2$ (event vs. process) ANOVA was performed on the mean numbers of parts of the nouns averaged across participants (see Table 2). Two main effects were revealed. There was a significant difference in the numbers of parts between physical activities and mental activities, with more temporal parts listed for physical activities $(M=4.15)$ than for mental activities $(M=2.84)\left[F(1,20)=18.88, M S_{\mathrm{e}}=0.54, p<\right.$ $.001]$. There was also a significant difference in the numbers of parts between events and processes, with more temporal parts listed for events $(M=4.17)$ than for processes $(M=$ 2.82) $\left[F(1,20)=20.19, M S_{\mathrm{e}}=0.54, p<.001\right]$. The test also indicated a significant interaction between physicality and aspect $\left[F(1,20)=5.47, M S_{\mathrm{e}}=0.54, p=.03\right]$.

Activities presented as gerunds. The ANOVA on the mean numbers of parts generated for gerundive terms revealed a consistent pattern: two main effects and an interaction effect $\left[F(1,20)=7.04, M S_{\mathrm{e}}=0.16, p=.02\right.$, for physicality; $F(1,20)=15.31, M S_{\mathrm{e}}=0.16, p<.001$, for aspect; and $F(1,20)=5.43, M S_{\mathrm{e}}=0.16, p=.03$, for their interaction]. Participants generated more parts for physical activities $(M=3.38)$ than for mental activities $(M=$ $3.01)$, and more parts for events $(M=3.55)$ than for processes $(M=2.92)$.

These findings indicate that both physicality and aspect influence the temporal structure of an activity. Physical activities had more temporal parts than did mental activities, and events had more temporal parts than did processes. In addition, the difference between physical events and mental events was more pronounced than that between physical processes and mental processes. This may suggest that aspect differences are quite salient for physical activities, but less so for mental activities. Alternatively, participants may have had more difficulties generating parts for mental activities than for physical activities because mental activities were more abstract. To further test the effects of physicality and aspect on the structure of activities, we conducted another experiment with different measures of homogeneity.

\section{EXPERIMENT 3}

The number of listed temporal parts is only one aspect of activity homogeneity. Even an activity that is described

Table 2

Mean Numbers of Parts of Physical Events, Physical Processes, Mental Events, and Mental Processes in Experiment 2

\begin{tabular}{cccccc}
\hline & \multicolumn{2}{c}{ Events } & & \multicolumn{2}{c}{ Processes } \\
\cline { 2 - 3 } \cline { 5 - 6 } & $M$ & $S D$ & & $S D$ \\
\hline Nouns & & & & \\
Physical & 5.18 & 1.04 & & 3.12 & 0.76 \\
Mental & 3.17 & 0.51 & & 2.52 & 0.50 \\
Gerunds & & & & \\
Physical & 4.16 & 0.44 & & 2.90 & 0.41 \\
Mental & 3.28 & 0.49 & & 2.79 & 0.26 \\
\hline
\end{tabular}

with many temporal parts can be perceived as homogeneous if these parts are relatively similar to each other; likewise, an activity with few temporal parts can be perceived as heterogeneous if these parts have distinctive characteristics. Experiment 3 examined the similarity of the temporal parts listed in the previous experiment across the four types of activity. To this end, a property listing approach was adapted from Rips and Estin's (1998) study. The numbers of common and distinctive properties listed for the temporal parts were used as the measures for homogeneity. As in the last experiment, the prediction was that the distinctiveness of temporal parts would be influenced by temporal aspect, and that the effect of physicality would therefore either emerge as a smaller factor than assumed previously or disappear.

\section{Method}

Participants. One hundred twenty participants who had not participated in any of the previous experiments were recruited from undergraduate introductory psychology classes at NIU. Most of these students were freshmen and sophomores. Students obtained extra course credit for their participation in this experiment.

Materials. The 24 activity terms (nouns) from Experiment 2 were used in this experiment. The reason that only nouns were used in this experiment was that noun stimuli and gerund stimuli provided very comparable results in Experiment 2. Following Rips and Estin's (1998) approach for each activity, the part listed most frequently for each activity in Experiment 2 was chosen to be evaluated in this experiment. If more than one part of an activity had the highest frequency - a rare occurrence - a part was randomly selected from these most frequent parts. The complete list of activities and their most frequent parts is presented in Appendix B.

Design. The 24 activity terms and the selected parts were divided into three sets to prevent fatigue effects among participants. Each set included two mental events, two physical events, two mental processes, and two physical processes, along with their parts, in random order. Each set was presented with two different instructions (common property listing or distinctive property listing), yielding altogether six conditions. Participants were randomly assigned into one of the six conditions. That is, physicality and temporal aspect were varied within subjects; the instruction type was varied between subjects.

Procedure. The experiment used a paper-and-pencil procedure. All materials were presented in booklets, with one activity and its corresponding part appearing at the top of each page. Participants recorded their responses in writing below the activity and its part.

Participants in the common property condition and the distinctive property condition received different instructions. For example, participants were asked to "list the properties of answering questions that are true of all other parts of an interview" in the common property condition. In contrast, participants were asked to "list the properties of answering questions that are true of no other parts of an interview" in the distinctive property condition.

\section{Results and Discussion}

Only properties listed by at least 2 participants for a part were included in the analyses. Doing so was expected to eliminate idiosyncratic responses and to increase the reliability of the analysis. Furthermore, synonymous properties were counted as the same property for a part. Examples of the properties can be found in Appendix C. The numbers of distinctive and of common properties were averaged across participants for each part of an activity.

Distinctive properties. A 2 (physical/mental) $\times 2$ (event/process) ANOVA examined the effects of physical- 
ity and aspect on the number of distinctive properties. The more homogeneous an activity is, the less distinct its parts should be. On average, the parts of physical activities had more distinctive properties $(M=2.75)$ than did the parts of mental activities $(M=2.00)\left[F(1,20)=22.91, M S_{\mathrm{e}}=\right.$ $0.15, p<.001]$. Furthermore, the parts of events had more distinctive properties $(M=2.66)$ than did the parts of processes $(M=2.09)\left[F(1,20)=12.88, M S_{\mathrm{e}}=0.15, p<\right.$ .01]. There was no interaction between these two factors $(F<1)$. The effect size of physicality was slightly higher $\left(\eta^{2}=.53\right)$ than that of temporal aspect $\left(\eta^{2}=.39\right)$.

Common properties. A second ANOVA with the same factors was carried out on the number of common properties. There were no significant differences in homogeneity between different activity types as measured by common property (all $F$ values were smaller than 1 ). That is, their parts had similar amounts of commonalities (Table 3). This is consistent with Rips and Estin's (1998) findings that participants did not list different amounts of common properties for physical and mental activities. One possible explanation is that very limited numbers of common properties were listed for all items. Many fewer common properties than distinctive properties were listed in this experiment $\left[F(1,23)=13.07, M S_{\mathrm{e}}=0.30, p<\right.$ $.001]$. This may reflect different task strategies: When participants listed distinctive properties, they included sub-phases of a part as well as abstract properties of the part; when participants listed common properties, they only used abstract properties, which are more difficult to describe than are sub-phases.

Distinctive versus common properties. Both physicality and temporal aspect were associated with homogeneity of activities, as measured by distinctive properties listed for their parts. However, the distinctive property measure alone is not a reliable measure for homogeneity, because two parts with the same distinctive property scores may have different common property scores. For example, the part of one activity may have as many common properties as distinctive properties, and the part of another may have only one-fifth as many common properties as distinctive properties. Plausibly, the latter part should be perceived as more distinct, because the distinctive properties would outweigh the common properties. Likewise, two activities may be similarly homogeneous, even if the part of one activity possesses fewer distinctive properties than the part of the other, if the part at the same time possesses fewer common properties than the part of the other.

Table 3

Mean Numbers of Distinctive and Common Properties of the Parts in Experiment 3

\begin{tabular}{llllll}
\hline & \multicolumn{2}{c}{ Events } & & \multicolumn{2}{c}{ Processes } \\
\cline { 5 - 6 } & $M$ & $S D$ & & $M$ & $S D$ \\
\hline $\begin{array}{l}\text { Distinctive Properties } \\
\quad \text { Physical }\end{array}$ & 3.11 & 0.42 & & 2.39 & 0.52 \\
$\quad$ Mental & 2.20 & 0.31 & & 1.79 & 0.23 \\
$\quad \begin{array}{lllll}\text { Common Properties } \\
\quad \text { Physical }\end{array}$ & 1.92 & 0.82 & & 1.91 & 0.61 \\
$\quad$ Mental & 1.52 & 0.58 & & 1.86 & 0.37 \\
\hline
\end{tabular}

Intuitively, the parts of physical activities may be described as having relatively more distinctive and more common properties because they tend to involve concrete elements around which their parts can be divided (see, e.g., B. Tversky, Morrison, \& Zacks, 2002). Such elements may make the properties of the temporal phases easier to describe in words, and thus elongate the list of common and distinctive properties. In contrast, mental activities rarely involve objects, and thus their parts may only elicit few distinctive and common properties.

If this speculation is correct, participants should have listed more properties overall for physical activities than for mental activities. This was supported by a two-way ANOVA on the sum of distinctive properties and common properties. On average, significantly more properties were listed for a physical activity part $(M=4.67)$ than for a mental activity part $(M=3.68)\left[F(1,20)=9.52, M S_{\mathrm{e}}=0.61, p<\right.$ $.01]$. This finding mirrors data for concrete versus abstract concept pairs (e.g., Markman \& Gentner, 1993). Temporal aspect did not elicit significantly different numbers of properties $(M=4.38$ for event and $M=3.98$ for process) $\left[F(1,20)=1.56, M S_{\mathrm{e}}=0.01, p=.23\right]$. Table 4 (top) lists the total numbers of properties for all activity types.

In short, to the extent that physicality affects the total number of generated properties, whether common or distinctive, it may artificially increase the homogeneity differences as measured by the distinctive property score alone. Since this appears to be the case, a measure that takes into account both distinctive and common properties may be a more adequate measure for the distinctiveness of temporal parts across different types of activity (see also A. Tversky, 1977). Such a measure may reveal that physicality does not have quite so big an effect on homogeneity. Therefore, a corrected distinctiveness measure was constructed by subtracting common properties from distinctive properties. The higher this score is, the more distinct the temporal part is from other parts of the activity.

Table 4 (middle) shows this average corrected distinctiveness measure, distinctive properties - common properties, for each activity type. A 2 (physical vs. mental) $\times$ 2 (event vs. process) ANOVA on this score showed that parts of events were more distinct $(M=0.94)$ than parts of processes $(M=0.21)\left[F(1,20)=7.16, M S_{\mathrm{e}}=0.45\right.$, $p=.02]$. Thus, consistent with the previous experiments, processes emerged as significantly more homogeneous than events. The main effect of physicality was marginally significant $\left[F(1,20)=3.66, M S_{\mathrm{e}}=0.45, p=.07\right]$. The interaction effect was not significant $(F<1)$.

Some authors suggest that a ratio score is a more appropriate way to measure relative similarity (e.g., Bush \& Mosteller, 1951; Gregson, 1975), so a ratio score ([distinctive properties - common properties]/common properties) was computed. To rectify normality violation, a natural log transformation was performed on the ratio score (normality test, $p=.06$ ). The ANOVA on this transformed ratio score revealed rather comparable results: Only the effect of temporal aspect was significant $\left(F=5.43, M S_{\mathrm{e}}=0.02\right.$, $p=.02)$. The main effect of physicality did not reach significance $\left(F=1.49, M S_{\mathrm{e}}=0.02, p=.24\right)$. The interaction effect was not significant $(F<1)$, either. 
Table 4

Total Score, Difference Score, and Ratio Score of Distinctive and Common Properties in Experiment 3

\begin{tabular}{cccccc}
\hline & \multicolumn{2}{c}{ Events } & & \multicolumn{2}{c}{ Processes } \\
\cline { 2 - 3 } \cline { 5 - 6 } & $M$ & $S D$ & & $M$ & $S D$ \\
\hline Total & & & & \\
Physical & 5.04 & 0.91 & & 4.31 & 1.04 \\
Mental & 3.72 & 0.61 & 3.65 & 0.41 \\
Difference & & & & \\
Physical & 1.19 & 0.95 & 0.48 & 0.44 \\
Mental & 0.69 & 0.70 & & 0.07 & 0.46 \\
Ratio & & & & \\
Physical & 1.02 & 0.14 & 0.93 & 0.07 \\
Mental & 0.99 & 0.17 & 0.83 & 0.11
\end{tabular}

Note-The ratio score was transformed to rectify violation of the normality principle. It was computed as [distinctive properties - common properties]/common properties.

\section{EXPERIMENT 4}

Experiment 4 aimed to address two concerns regarding the methodology of Experiment 3. First, Experiment 3 quantified the differences in homogeneity by comparing the numbers of listed features, both distinctive and common features. However, as one can imagine, some features can be essential to the corresponding activity; others may not. For example, being together is probably more essential to a date than knocking on the door; breaking down seems more essential to the process of decomposition than smell. Therefore, each feature should not be regarded as equally important. Instead, it is more reasonable to attach different features with different weights when being evaluated together. Counting numbers of its features provides only a rough estimate on how distinct a part is within the whole activity, and thus the difference in numbers of listed features only a rough estimate on the difference of homogeneity. To obtain a more accurate measure, in Experiment 4 we instructed participants to rate the perceived homogeneity of activities.

The second concern with Experiment 3 is that only the most frequently listed parts were selected for the feature listing task. One could argue that, even though those parts could be considered the most typical parts of their corresponding activities, they could be different in terms of how easy they are to be segmented from other parts of the corresponding activities. For example, a part may be easier to differentiate if it is the initial or the final phase of the activity than if it is one of the intermediate phases of the activity. Therefore, comparison of the distinctiveness of the most frequently listed parts among different activities may not reveal the difference in overall homogeneity among these activities. To solve this problem, Experiment 4 tested homogeneity using the most frequently listed part (as in Experiment 3), as well as randomly sampled parts for each activity. It was hypothesized that both physicality and aspect affected the homogeneous nature of an activity. That is, the parts of physical activities would receive higher ratings on distinctiveness than the parts of mental activities; the parts of events would receive higher ratings on distinctiveness than the parts of processes. However, given the findings from Experiment 3, aspect may have a stronger impact on homogeneity than does physicality.

\section{Method}

Participants. Seventy-two participants who had not participated in any of the previous experiments were recruited from undergraduate psychology classes at NIU. Students received course credit for their participation in this experiment.

Materials. For each of the 24 activities, one part was randomly sampled from among those listed by the participants in Experiment 2 . The most frequently listed parts were excluded from this sampling process. As discussed before, for some activities, especially processes, very few parts were generated. In the case of an activity with only one part generated, a different expression of that part was chosen from among the actual responses. The 24 most frequently listed parts, as a comparison, were also used in this experiment. All materials are listed in Appendix B.

Procedure. Participants were randomly assigned into two groups, 36 in each group. Both groups were presented a sheet of paper with the 24 activities in a random order. One part of the activity was presented along with the activity. The first group received the parts listed most frequently by participants in Experiment 2 (i.e., the same parts as in Experiment 3). The second group received the parts randomly selected from the responses in Experiment 2. Both groups were instructed to rate, on a 6.0 scale, how easy it was to differentiate the given part from other (not presented) parts of the corresponding activity. On the scale, 1 indicated extremely difficult to differentiate, and 6 indicated extremely easy to differentiate.

\section{Results and Discussion}

Distinctiveness of the most frequent parts. The ratings for the most frequent parts were averaged across participants. The mean ratings were entered into a 2 (physical/ mental) $\times 2$ (event/process) ANOVA. Replicating the effects from Experiment 3, two main effects were revealed in this analysis (Table 5). On average, the parts of physical activities $(M=4.20)$ were rated as easier to distinguish than the parts of mental activities $(M=3.77)[F(1,20)=$ $\left.12.75, M S_{\mathrm{e}}=0.08, p<.01\right]$. The parts of events $(M=$ 4.26) were rated as easier to distinguish than the parts of processes $(M=3.71)\left[F(1,20)=19.84, M S_{\mathrm{e}}=0.08\right.$, $p<.001$ ] . Consistent with Experiment 3, the effect size of physicality (.39) was somewhat smaller than the effect size of aspect (.50). The interaction was not significant $(F<1)$.

Distinctiveness of the randomly sampled parts. The ratings for the randomly sampled parts were averaged across participants. An ANOVA with the same factors as the previous one also showed two main effects (Table 5). On average, the parts of physical activities $(M=4.03)$ were rated as easier to distinguish than the parts of mental activities $(M=3.60)\left[F(1,20)=5.17, M S_{\mathrm{e}}=0.21, p=\right.$ $.03]$; the parts of events $(M=4.13)$ were rated as easier to distinguish than the parts of processes $(M=3.50)$ $\left[F(1,20)=11.43, M S_{\mathrm{e}}=0.21, p<.01\right]$. Again, the effect size of physicality (.21) was somewhat smaller than the effect size of aspect (.36). The interaction between the two factors was not significant $(F<1)$.

In summary, the ratings for both most frequent parts and randomly sampled parts replicated the results from Experiment 3, indicating that both factors, physicality and aspect, influence the perceived homogeneity of the activities. Physical activities appeared to be less homogeneous 
Table 5

Mean Ratings of the Most Frequent Parts and the Randomly Sampled Parts in Experiment 4

\begin{tabular}{lccccc}
\hline & \multicolumn{2}{c}{ Events } & & \multicolumn{2}{c}{ Processes } \\
\cline { 2 - 3 } \cline { 5 - 6 } & $M$ & $S D$ & & $M$ & $S D$ \\
\hline Most Frequent Parts & & & & \\
$\quad$ Physical & 4.47 & 0.41 & & 3.94 & 0.26 \\
$\quad$ Mental & 4.05 & 0.28 & & 3.49 & 0.21 \\
$\quad$ Randomly Sampled Parts & & & & \\
$\quad$ Physical & 4.40 & 0.49 & & 3.66 & 0.39 \\
$\quad$ Mental & 3.87 & 0.61 & 3.33 & 0.29 \\
\hline
\end{tabular}

than mental activities; events appeared to be less homogeneous than processes.

\section{GENERAL DISCUSSION}

This study examined the difference between the temporal structures of physical and mental events and processes. The main question was whether physicality accounted for any variance in homogeneity after aspect was taken into account. The experiments showed that the temporal structure of activity, as measured by the numbers of parts, the numbers of the properties of the parts, and the distinctiveness ratings of the parts, is associated with both physicality and temporal aspect.

Rips and Estin (1998) discussed compositional differences between objects and events. Objects can easily be distinguished into heterogeneous parts, whereas events are more difficult to break into parts. Often, different temporal phases of an event share situational elements, such as people and objects. This further contributes to the homogeneity of the parts. Our findings further show that a distinction can be made between events and processes; that objects are more heterogeneous than events; and that events are more heterogeneous than processes. Parts of processes listed in this study were usually synonyms of the processes, whether the processes were physical or mental. This additional distinction goes back to Vendler's (1967) theory, according to which any part of a process is of the same nature as the whole. Another linguist, Comrie (1976), also argued the aspect distinction between event and process, and pointed out that this distinction mirrors the linguistic distinction between perfective and imperfective. Although both are dynamic situations, events are viewed as complete, whereas processes are viewed as in progress. Our data strongly support the psychological validity of these linguistic theories on the distinction in temporal aspect.

The results of this study show quite clearly that physical events are less homogeneous than physical processes. Barbara Tversky, Zacks, and Lee (2004) argued that physical events may involve more objects that can serve as dividing points than do physical processes. One may question what the distinction between mental events (e.g., deciding) and mental processes (e.g., thinking) is based on in this research. One basis for this distinction lies in the presence of change. The activities judged as mental events involved abrupt internal changes within the sequence. For example, approval was judged as a men- tal event, and can be described as an abrupt shift from evaluating relevant information to making the final decision of approval. In contrast, activities that were judged as mental processes (e.g., imagination) did not generally contain such a moment of change. The norming study for Experiment 2 suggests that individuals are indeed sensitive to that difference. Our judges reliably distinguished mental processes $(79.2 \%$ agreement rate) from mental events (83.3\% agreement rate). In Experiments 2 and 3 , little difference was observed between mental events and processes when the numbers of parts and properties served as the measure. However, we obtained some evidence for the distinction measured by rating scores in Experiment 4. Parts of mental events were rated as more distinct than parts of mental processes $[t(10)=3.97, p<$ .01 , for the most frequent parts; $t(10)=1.92, p=.08$, for the randomly selected parts]. Importantly, this suggests that future studies on the structure of mental activities need to employ alternative methods rather than part and property listing. As indicated earlier, fewer parts and properties were listed for mental activities than for physical activities; therefore, differences between the mental events and processes may have been covered up by the low variance, and participants' rating may be a more sensitive measure.

A limitation of this research is that activity terms of different types may have been unwittingly selected from different category levels. As Rips and Estin (1998) pointed out, it is dubious whether superordinate object categories (e.g., furniture) and superordinate event categories (e.g., cognitive processes) have equivalent characteristics, such as size and commonalities. Because it is difficult to align abstraction levels across activity domains, concepts utilized in our study and by Rips and Estin may have been selected from different levels of abstraction. As a consequence, it is possible that the differences between objects, events, and processes in studies like this one may be exaggerated or underestimated. For example, Morris and Murphy (1990) found that social events were more distinguishable at the highest abstraction level than at lower levels (including the basic level), whereas objects were most distinguishable at the basic level.

In conclusion, the role of physicality in homogeneity was a robust factor in the analysis even after factoring in the role of aspect, which extends and clarifies Rips and Estin's (1998) results. Additionally, aspect emerged as a critical factor for concept homogeneity. We obtained some evidence for the distinction between both physical events versus processes and mental events versus processes.

\section{AUTHOR NOTE}

Some of the data reported in this article were collected as part of the Master's thesis of the first author. Correspondence concerning this article should be addressed to $\mathrm{X}$. $\mathrm{Xu}$, School of Behavioral Sciences and Education, Pennsylvania State University at Harrisburg, Middletown, PA 17057 (e-mail: xuxu@psu.edu).

\section{REFERENCES}

Boltz, M. G. (1998). The processing of temporal and nontemporal information in the remembering of event durations and musical struc- 
ture. Journal of Experimental Psychology: Human Perception \& Performance, 24, 1087-1104.

Bush, R. R., \& Mosteller, F. (1951). A model for stimulus generalization and discrimination. Psychological Review, 58, 413-423.

ComrIE, B. (1976). Aspect: An introduction to the study of verbal aspect and related problems. Cambridge: Cambridge University Press.

Gregson, R. A. M. (1975). Psychometrics of similarity. New York: Academic Press.

Magliano, J. P., Miller, J., \& ZwaAn, R. A. (2001). Indexing space and time in film understanding. Applied Cognitive Psychology, 15, 533-545.

Markman, A. B., \& Gentner, D. (1993). Splitting the differences: A structural alignment view of similarity. Journal of Memory \& Language, 32, 517-535.

Morris, M. W., \& Murphy, G. L. (1990). Converging operations on a basic level in event taxonomies. Memory \& Cognition, 18, 407-418.

Newtson, D. (1973). Attribution and the unit of perception of ongoing behavior. Journal of Personality \& Social Psychology, 28, 28-38.
RiPs, L. J., \& Estin, P. A. (1998). Components of objects and events. Journal of Memory \& Language, 39, 309-330.

Schank, R. C., \& Abelson, R. P. (1977). Scripts, plans, goals, and understanding: An inquiry into human knowledge structures. Hillsdale, NJ: Erlbaum.

Tversky, A. (1977). Features of similarity. Psychological Review, 84, 327-352.

Tversky, B., Morrison, J. B., \& Zacks, J. (2002). On bodies and events. In A. N. Meltzoff \& W. Prinz (Eds.), The imitative mind: Development, evolution, and brain bases (pp. 221-232). Cambridge: Cambridge University Press.

TVersky, B., Zacks, J. M., \& LeE, P. (2004). Events by hands and feet. Spatial Cognition \& Computation, 4, 5-14.

Vendler, Z. (1967). Linguistics in philosophy. Ithaca, NY: Cornell University Press.

ZACKS, J. M., \& TVERSKY, B. (2001). Event structure in perception and conception. Psychological Bulletin, 127, 3-21.

\begin{tabular}{ll}
\multicolumn{2}{c}{ APPENDIX A } \\
Stimulus Categories in Rips and Estin's (1998) Study \\
\hline \multicolumn{1}{c}{ Script } & Mental Activity \\
\hline Writing a letter & Reading \\
Checking out books & Reasoning \\
Getting a suntan & Planning \\
Going to restaurants & Conceptualizing \\
Catching a plane & Dreaming \\
Taking a photograph & Contemplating \\
Xeroxing a page & Having emotions \\
Going to movies & Imagining \\
Sending a gift & Realizing \\
Going on vacation & Loving \\
Cashing a check & Deciding \\
Doing the dishes & Remembering \\
\hline
\end{tabular}


Stimulus Activities From Experiment 2 and the

Corresponding Parts Presented As Stimuli in Experiment 4

\begin{tabular}{|c|c|c|}
\hline Activity & Most Frequent Part & Randomly Sampled Part \\
\hline \multicolumn{3}{|c|}{ Physical Events } \\
\hline Funeral & Viewing the body & Traveling to the burial place \\
\hline Date & Picking up someone/being picked up by someone & Talking to one's company \\
\hline Party & Mingling & Having fun \\
\hline Robbery & Taking what is wanted & Breaking into a place \\
\hline Concert & Buying tickets & Introducing the performers \\
\hline Interview & Answering questions & Getting dressed nicely \\
\hline \multicolumn{3}{|c|}{ Physical Processes } \\
\hline Acceleration & Going faster than before & Increasing speed \\
\hline Investigation & Searching for clues & Putting information together \\
\hline Development & Growing & Reaching the next stage \\
\hline Exploration & Discovering something new & Searching for place \\
\hline Decomposition & Rotting & Breaking down \\
\hline Management & Supervising others & Reporting things to upper level \\
\hline \multicolumn{3}{|c|}{ Mental Events } \\
\hline Déjà vu & Experiencing something & Remembering when it has happened \\
\hline Judgment & Comparing facts or arguments & Thinking about the possible outcomes \\
\hline Debate & Forming a topic & Gathering information for the topic \\
\hline Recognition & Seeing something & Remembering from the past \\
\hline Approval & Evaluating & Looking at the presentation \\
\hline Realization & Noticing something & Understanding things \\
\hline \multicolumn{3}{|c|}{ Mental Processes } \\
\hline Imagination & Creating something in mind & Thinking of new, unique things \\
\hline Elaboration & Expanding statements & Adding more to the statement \\
\hline Conceptualization & Forming a concept & Understanding from all angles \\
\hline Reflection & Thinking through & Thinking in depth \\
\hline Contemplation & Weighing up strengths and weaknesses & Thinking over all the details \\
\hline Recollection & Remembering & Searching for the memory \\
\hline
\end{tabular}

APPENDIX C

Example Properties Listed for the Parts of Activities in Experiment 3

\begin{tabular}{|c|c|c|}
\hline Part & Common Property & Distinct Property \\
\hline Viewing the body (Funeral) & Sad & Walking in line \\
\hline Picking up someone/being picked up by someone (Date) & Being together & Knocking on the door \\
\hline Mingling (Party) & Walking around & Talking \\
\hline Taking what is wanted (Robbery) & By force & Satisfied \\
\hline Buying tickets (Concert) & Cost & Ticket booth \\
\hline Answering questions (Interview) & Nervous & Thinking of answers \\
\hline Going faster than before (Acceleration) & Gaining speed & Quicker \\
\hline Searching for clues (Investigation) & Alert & Looking around \\
\hline Growing (Development) & Getting bigger & Gaining weight \\
\hline Discovering something new (Exploration) & Looking around & Surprised \\
\hline Rotting (Decomposition) & Smell & Breaking down \\
\hline Supervising others (Management) & Watching over & Responsible \\
\hline Experiencing something (Déjà vu) & Feeling repetition & New experience \\
\hline Comparing facts or arguments (Judgment) & Thought process & Information \\
\hline Forming a topic (Debate) & Interesting topic & Deciding what to talk about \\
\hline Seeing something (Recognition) & Familiar objects & Looking \\
\hline Evaluating (Approval) & Thinking & Analyzing \\
\hline Noticing something (Realization) & Seeing something & Observant \\
\hline Creating something in mind (Imagination) & Creative & Images \\
\hline Expanding statements (Elaboration) & More detail & More information \\
\hline Forming a concept (Conceptualization) & Thinking & Thinking of an idea \\
\hline Thinking through (Reflection) & Remembering & Past experience \\
\hline Weighing up strengths and weaknesses (Contemplation) & Strengths and weaknesses & Compare and contrast \\
\hline Remembering (Recollection) & Thinking about it & Flashback \\
\hline
\end{tabular}

(Manuscript received February 3, 2005;

revision accepted for publication January 28, 2006.) 